Document downloaded from:

http://hdl.handle.net/10251/166270

This paper must be cited as:

Busolo, M.; Torres-Giner, S.; Prieto, C.; Lagaron, JM. (2019). Electrospraying assisted by pressurized gas as an innovative high-throughput process for the microencapsulation and stabilization of docosahexaenoic acid-enriched fish oil in zein prolamine. Innovative Food Science \& Emerging Technologies. 51:12-19. https://doi.org/10.1016/j.ifset.2018.04.007

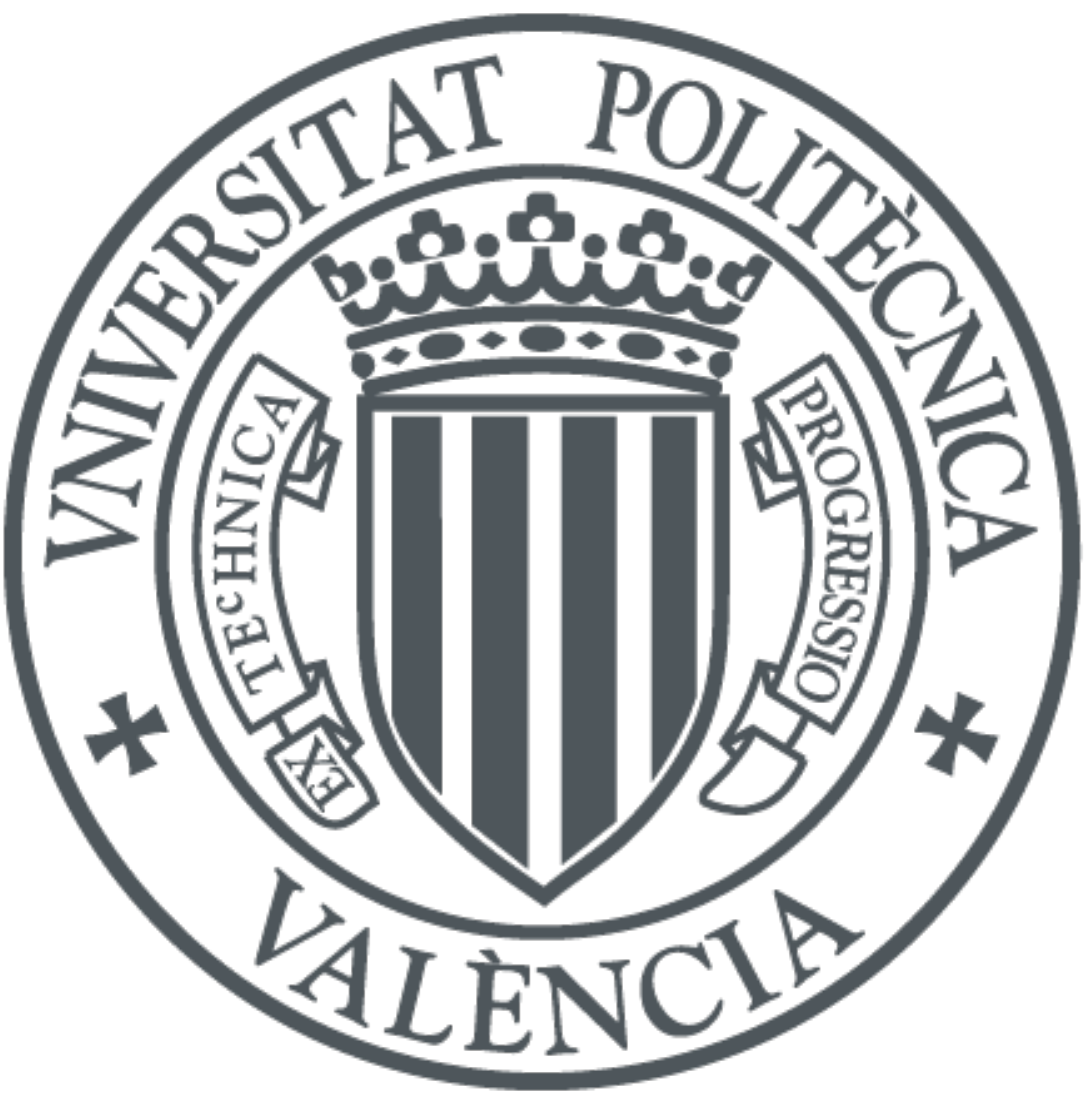

The final publication is available at

https://doi.org/10.1016/j.ifset.2018.04.007

Copyright Elsevier

Additional Information 


\title{
Electrospraying Assisted by Pressurized Gas as an Innovative High-throughput Process for the Microencapsulation and Stabilization of Docosahexaenoic Acid- enriched Fish Oil in Zein Prolamine
}

\author{
M. A. Busolo ${ }^{1,2}$, S. Torres-Giner ${ }^{1}$, C. Prieto $^{1}$ and J.M. Lagaron ${ }^{1}$ \\ ${ }^{1}$ Novel Materials and Nanotechnology Group, Institute of Agrochemistry and Food \\ Technology (IATA), Spanish National Research Council (CSIC). Calle Catedrático \\ Agustín Escardino Benlloch 7, 46980 Paterna, Valencia, Spain.
}

${ }^{2}$ Bioinicia S.L. Calle Algepser 65, nave 3, 46980 Paterna, Valencia, Spain.

\section{Abstract}

Zein, a prolamine obtained from maize, was employed to encapsulate a fish oil highly enriched with docosahexaenoic acid (DHA) by an innovative process termed electrospraying assisted by pressurized gas (EAPG). This technology combines high electric voltage with pneumatic spray to yield a high-throughput encapsulation process. Semi-spherical zein flowable capsules with mean sizes of $1.4 \mu \mathrm{m}$ containing the DHAenriched fish oil were produced by EAPG from inert ethanol solutions at room conditions, presenting a high encapsulation efficiency. The oxidative stability tests carried out in the zein microcapsules obtained by EAPG showed that the DHA-enriched fish oil was efficiently protected over storage time. Sensory tests were also performed on fortified reconstituted milk with the freshly prepared zein/DHA-enriched fish oil microcapsules, suggesting negligible oxidation effects after 45 days. The results described herein indicate that EAPG is a promising innovative high-throughput electrospraying-based methodology for the encapsulation of bioactives and, therefore, the resultant DHAenriched fish oil containing microcapsules can be industrially applied for the formulation of fortified foods.

Keywords: Zein; DHA; Fish Oil; Electrospraying; Encapsulation; Nutraceuticals 


\section{INTRODUCTION}

The omega-3 polyunsaturated fatty acids (PUFAs), namely eicosapentaenoic acid (EPA) and docosahexaenoic acid (DHA), are mainly found in extracted fish oils from marine fish. These PUFAs are known to exert a variety of health benefits, including hypotriglyceridemic and anti-inflammatory effects, besides antihypertensive, anticancer, antioxidant, anti-depression, antiaging, and antiarthritis effects, as supported by recent studies (Arbabi, Baharuldin, Moklas, Fakurazi, \& Muhammad, 2014; Park, Kwon, Han, Hahm, \& Kim, 2013; Ruxton, Reed, Simpson, \& Millington, 2004; Siriwardhana, Kalupahana, \& Moustaid-Moussa, 2012; Vaughan, Hassing, \& Lewandowski, 2013; Zainal, et al., 2009). PUFAs also play crucial roles during growth and development in children as well as in heart, brain, and eye health in adults. Previous research based on non-human studies suggests that intake above normal nutritional requirements might modify the risk/course of a number of diseases (Ruxton, et al., 2004). The widely investigated multiple health benefits of PUFAs encourage their consumption, especially for low-fish dietary sources, but these have also fueled much of the present research to determine mechanisms whereby DHA may serve as a nutraceutical (Sun, et al., 2017). Nutraceuticals are dietary supplements that deliver a concentrated form of a biologically active component, typically referred as bioactive, from a foodstuff to enhance health in dosages that exceed those that could be obtained from regular food intake (Zeisel, 1999). The resultant functional foods currently represent an important trend in a multi-niche market as these provide consumers with an alternative way to achieve a healthy lifestyle that differs from conventional healthy diets (Ayelén Vélez, Cristina Perotti, Santiago, María Gennaro, \& Hynes, 2017). There are many types of commercially available food products already fortified with omega-3 PUFAs, either supplemented via animal feed or manufactured from enriched ingredients, including milk and dairy products, eggs and meat, bread and bakery, cooking oil, jellies, beverages, chocolate, cereal bars, etc. (Ganesan, Brothersen, \& McMahon, 2014; Lopez-Huertas, 2010).

However, fish oils impart their typical fishy flavors when are directly added to foods. Moreover, their additional unpleasant odor and flavor, which result from their poor oxidative stability, limit severely their application as a nutraceutical for functional foods. Some pathways have been applied to stabilize fish oil such as addition of antioxidants to the bulk oil -which does not allow to remove unpleasant flavors-, emulsion-based delivery systems, and encapsulation (C. J. Barrow, Wang, Adhikari, \& Liu, 2013; Encina, Vergara, 
Giménez, Oyarzún-Ampuero, \& Robert, 2016; Prieto \& Calvo, 2017; Wang, Liu, Chen, \& Selomulya, 2016). Several methods have been used for DHA encapsulation, such as spray-drying (SD) process, freeze-drying (FD) process, coacervation, spray granulation (SG), emulsification, supercritical fluids, and electrospraying (Anwar, Weissbrodt, \& Kunz, 2010; C. J. Barrow, et al., 2013; Encina, et al., 2016; García-Moreno, et al., 2017; García-Moreno, et al., 2016; Moomand \& Lim, 2014; Pereira, Valentão, \& Andrade, 2014; Torres-Giner, Martinez-Abad, Ocio, \& Lagaron, 2010). The encapsulation efficiency, stability, and protection of DHA achieved by these techniques also depend on the composition of the encapsulation wall material. Depending on the process and the desired behavior of the product, a wide range of polymer materials have been used for encapsulation of fish and microalgal oils, for instance proteins such as caseinate, gelatin, zein, whey protein isolate (WPI), and soybean isolate (SI) or polysaccharides such as maltodextrin, pullulan, chitosan, and some blends of glucose syrup, cyclodextrins, pectin, xanthan, and lactose, among others (Aghbashlo, Mobli, Madadlou, \& Rafiee, 2012; Bakry, et al., 2016; C. Barrow, Van Diepen, Perrie, Curtis, Jin, \& Zhang, 2007; Chen, Wang, Zhang, Gao, Chen, \& Li, 2016; Encina, et al., 2016; Moomand, et al., 2014; Pereira, et al., 2014).

Among the different encapsulation technologies, SD process from oil-in-water $(\mathrm{o} / \mathrm{w})$ emulsions is currently employed to produce fish oil microencapsulated powders intended for food products (e.g. infant powder formulas, baked products, and beverages) (C. J. Barrow, et al., 2013; Encina, et al., 2016). SD has been employed to encapsulate different fish oils in a wide variety of biopolymers and proteins under different formulations and operational conditions, involving the dehydration of emulsion droplets in a heated chamber at $140-210^{\circ} \mathrm{C}$ (Encina, et al., 2016; Wang, et al., 2016). Indeed, temperature is an important processing variable because the raise of the inlet air temperature increases the extent of oxidative reactions (Anwar \& Kunz, 2011; Hogan, O'Riordan, \& O'Sullivan, 2003), which becomes more relevant under non-inert atmospheres. In spite of this, SD is the most common encapsulation method for fish oil due to its relatively low production costs and the scaling-up difficulties typically associated to other techniques. Encapsulation of fish oils has also been performed by FD process in soybean soluble polysaccharide, starch, WPI or chitosan as the wall materials. In particular, FD is based on the dehydration by sublimation of the ice fraction of frozen fish oil emulsions (Encina, et al., 2016; Heinzelmann, Franke, Jensen, \& Haahr, 2000). Some recent findings have shown that FD-microencapsulated fish oil was more susceptible to oxidation than that 
encapsulated in SD capsules due to the irregular and highly porous structure of the FD capsules, caused by emulsion destabilization during the FD process (Anwar, et al., 2011). Contrary to SD and FD processes, SG is a soft method that uses mild temperatures, up to $70{ }^{\circ} \mathrm{C}$, to evaporate water from emulsions. The thermal stability of SG-microencapsulated fish oil is favored by eliminating the heat-assisted oxidation factor, though the bigger particle size obtained may affect organoleptic properties of the final product (Anwar, et al., 2011; Anwar, et al., 2010). Supercritical fluid extraction (SFE) has also been proven as efficient to encapsulate omega-3 PUFA, with an encapsulation efficiency similar to that generated by conventional solvent evaporation and high control on the particle size (Prieto, et al., 2017). However, SFE process requires high capital cost and it is limited to the encapsulation of lipophilic compounds.

Electrohydrodynamic processing (EHDP), including both electrospinning and electrospraying techniques, is an emerging technology that has been particularly applied for fish oil encapsulation (Krokida, 2017), among a wide range of bioactive substances (Chang, Stride, \& Edirisinghe, 2010; Eltayeb, Stride, Edirisinghe, \& Harker, 2016; Shams, Parhizkar, Illangakoon, Orlu, \& Edirisinghe, 2017; Torres-Giner, Pérez-Masiá, \& Lagaron, 2016). In particular, electrospraying is based on the application of a high electric field to a charged polymer solution to produce ultrathin droplets, which after solidification result in nano- and submicro-sized capsules (Tapia-Hernández, et al., 2015). This process has been already employed to obtain fish oil- or DHA-loaded nanocapsules made of dextran (García-Moreno, et al., 2017) and zein (Torres-Giner, et al., 2010), respectively. The use of zein, a prolamine isolated from maize that is also accepted as generally recognized as safe (GRAS), presents certain advantages due to its high hydrophobicity, biocompatibility, and film-forming properties (Zhang, et al., 2016) but it also has some limitations such as lack of solubility in water and its characteristic yellowish color. In regard to the latter limitation, some manufacturers offer now commercial whitened zein grades. Different advanced carrier systems based on zein (e.g. nano- and microcapsules, films, hydrogels, etc.) have displayed improved properties in terms of stability and protection of active substances, release, and delivery efficiency (Zhang, et al., 2016). Therefore, the performance and versatility of zein encourage to continue investigating its potential uses as encapsulating material.

In general, one of the main disadvantages of the electrospraying process in the food and food packaging industry has typically been its low productivity, habitually with a 
processing throughput of a few milliliters per hour per single emitter (Torres-Giner, 2011). Since more recently companies like Bioinicia S.L. (www.bioinicia.com) have commissioned plants for the contract manufacturing at an industrial scale of electrospinning and electrospraying processes. In this context, Hong et al. (2017) proposed to couple pressure and infusion gyration to increase production without increasing the cost of the process. An innovative encapsulation technique based on the combination of electrospraying with the pneumatic atomization process is, for the first time, here presented. This novel high-throughput technology, termed as electrospraying assisted by pressurized gas (EAPG), is based on the atomization of the polymer solution by a pneumatic injector using compressed air/gas that nebulizes within a high electric field. During this process, the solvent is evaporated at room temperature in an evaporation chamber and the encapsulated material is then collected as a free-flowing powder. In the present study, EAGP is applied to encapsulate a DHA-enriched fish oil in a zein matrix. The encapsulation efficiency and the oxidative stability over time of the zein capsules containing the DHA-enriched fish oil were analyzed as a function of temperature and relative humidity (RH). Finally, the resultant DHA-enriched capsules were used to enrich milk and the organoleptic properties of the fortified milk were evaluated to ascertain their application in food products.

\section{MATERIALS AND METHODS}

\subsection{Materials}

Zein from maize, grade Z3625, and hydrochloric acid $(\mathrm{HCl}) 37$ vol.-\% were both purchased from Sigma-Aldrich S.A. (Madrid, Spain). Highly DHA-enriched fish oil was supplied by K.D. Pharma Bexbatch GmbH (Bexbach, Germany) as KD-Pür ${ }^{\circledR}$ DHA800 TG. According to the manufacturer, its DHA content ranges between 83.7-87.2 wt.- $\%$. The fish oil was stored in an airtight container, protected from light at $5{ }^{\circ} \mathrm{C}$. Barium chloride dihydrate $\left(\mathrm{BaCl}_{2} \cdot 2 \mathrm{H}_{2} \mathrm{O}\right)$, iron (III) chloride hexahydrate $\left(\mathrm{FeCl}_{3} \cdot 6 \mathrm{H}_{2} \mathrm{O}\right)$, iron (II) sulphate heptahydrate $\left(\mathrm{FeSO}_{4} \cdot 7 \mathrm{H}_{2} \mathrm{O}\right)$, magnesium nitrate $\left(\mathrm{Mg}\left(\mathrm{NO}_{3}\right)_{2}\right)$, ammonium thiocyanate $\left(\mathrm{NH}_{4} \mathrm{SCN}\right)$, and barium sulfate $\left(\mathrm{BaSO}_{4}\right)$, all of them as reagent grades, were purchased from Panreac S.A. (Barcelona, Spain). 2,2,4-trimethylpentane, also known as isooctane or iso-octane, reagent grade, was provided by Scharlab S.A. (Barcelona, Spain). Food-grade ethanol 96 vol.-\% was purchased from Guinama S.L. (Valencia, Spain). The 
bottled drinking water and skim milk powder, both used in organoleptic tests, were provided by Nestlé S.A. (Barcelona, Spain).

\subsection{Preparation of zein/DHA solution}

The zein/DHA-enriched fish oil solution was prepared by slow addition of the DHAenriched fish oil to an ethanol solution at $85 \mathrm{wt} . \mathrm{\%}$ containing $4.5 \mathrm{wt.} . \%$ of zein under vigorous nitrogen bubbling at room temperature. The zein to DHA-enriched fish oil ratio was kept fixed at 2:1 (wt./wt.) based on our previous work (Sergio Torres-Giner et al. 2010). The solutions were homogenized by means of an ultraturrax impeller at 14,000 $\mathrm{rpm}$. The prepared solution was immediately processed under constant nitrogen bubbling to minimize DHA-enriched fish oil oxidation. A zein solution without fish oil was also prepared as the control sample following the same procedure.

\subsection{EAPG process}

The prepared zein/DHA-enriched fish oil solution was processed by EAPG using a patent pending Fluidnatek ${ }^{\mathrm{TM}}$ LE500 Capsultek $^{\mathrm{TM}}$ pilot-plant from Bioinicia S.L. (Valencia, Spain) (Lagaron, Castro, Galan, \& Valle, 2017). This pilot installation comprises an injection unit, a drying chamber, and a cyclonic collector as described in Lagaron, Castro, Galan, \& Valle, 2017. The experiments here were optimally performed bubbling continuously nitrogen into the zein/DHA solution at controlled ambient conditions, i.e. $25^{\circ} \mathrm{C}$ and $40 \% \mathrm{RH}$, which was then pumped at $10 \mathrm{ml} / \mathrm{min}$ to nebulizer that worked with an air pressure of $10 \mathrm{l} / \mathrm{min}$. The nebulizer is connected to an electric voltage of $20 \mathrm{kV}$ and the resultant solution droplets dried in their travel towards the collecting unit. The generated capsules were collected every $20 \mathrm{~min}$ in the cyclone and stored in flasks, under vacuum, at $-5{ }^{\circ} \mathrm{C}$ and protected from light to avoid oxidation.

\subsection{Characterization of capsules}

\subsubsection{Microscopy}

Morphology of the DHA-enriched fish oil containing capsules was analyzed by scanning electron microscopy (SEM) in a Hitachi S-4800 FE-SEM from Hitachi High Technologies Corp. (Tokyo, Japan) with an electron beam acceleration of $5 \mathrm{KV}$. The samples were coated with a gold/palladium layer prior to SEM analysis. Capsule 
diameters were determined using Image J Launcher v 1.41 and the data presented were based on measurements from a minimum of 20 SEM micrographs.

Optical fluorescence microscopy was performed to ascertain the encapsulation and distribution of the DHA-enriched fish oil in the electrosprayed zein capsules. The optical microscopy images were acquired with an ECLIPSE E800 from Nikon (Kanagawa, Japan) equipped with a capture camera DXM1200F-Nikon, using a 40x objective. Fluorescence was measured with a UV-4A cyan filter. Excitation and emission wavelength ranges were $330-380 \mathrm{~nm}$ and $>420 \mathrm{~nm}$, respectively.

\subsubsection{Encapsulation efficiency}

Encapsulation efficiency was measured to estimate the capacity of the electrosprayed zein capsules to retain DHA-enriched fish oil inside the capsule. This was assessed by measuring the re-solubilization of the oil under a gentle surface washing method (GarcíaMoreno, et al., 2017; Moomand, et al., 2014). To this end, $25 \mathrm{mg}$ of capsules were placed in a glass tube with $5 \mathrm{ml}$ iso-octane, gently stirred and soaked for $1 \mathrm{~min}$. The mixture was then filtered and the absorbance of the filtrate was measured at $285 \mathrm{~nm}$ in a UV4000 spectrophotometer from Dinko S.A. (Barcelona, Spain). Standard solutions made of DHA-enriched fish oil and iso-octane at $0.1-0.5 \mathrm{mg} / \mathrm{ml}$ were used to build a calibration curve $\left(\mathrm{R}^{2}=0.99\right)$, from which the amount of DHA-enriched fish oil present in the liquid was determined. The encapsulation efficiency was then calculated as follows:

$$
\text { Efficiency }(\%)=[(A-B) / A] \cdot 100
$$

where $A$ is the theoretical amount of DHA-enriched fish oil and $B$ is the free amount of DHA-enriched fish oil detected in the supernatant. Measurements were carried out in triplicate. It should be noted that while this method has been widely applied in the existing literature, it has been typically applied to much higher particle sizes than obtained here and hence it may not be accurate enough for the very small particles sizes of the nonwater soluble zein used here. Thus, this method may facilitate extraction of oil from near the surface and not only necessarily from the particle surface.

\subsection{Peroxide Value determination}


Peroxide Value (PV) was used to analyze the oxidative stability of the DHA-enriched fish oil under different storage conditions. This was based on the principle that lipid peroxides are able to oxidize $\mathrm{Fe}^{2+}$ to $\mathrm{Fe}^{3+}$, and oxidation can be therefore spectrophotometrically quantified by means of ferric ion complexation with thiocyanate (Shantha \& Decker, 1994; Woods \& Mellon, 1941). For this, free DHA-enriched fish oil and zein/DHAenriched fish oil capsules were stored in glass desiccators at the conditions displayed in the Table 1. Different RH conditions were achieved by means of silica gel or a $\mathrm{Mg}\left(\mathrm{NO}_{3}\right)_{2}$ saturated solution, which provided RH values of $0 \%$ and $54 \%$, respectively. However, when vacuum was obtained within the desiccator containing a saturated solution of $\mathrm{Mg}\left(\mathrm{NO}_{3}\right)_{2}$, the $\mathrm{RH}$ increased from $54 \%$ to $65 \% \mathrm{RH}$ as measured by a hygrometer. PVs were determined for up to 45 days by following ISO 3976:1977 - Anhydrous milk fat: Determination of peroxide value - adapted from the International Dairy Federation (IDF) (Partanen, Raula, Seppänen, Buchert, Kauppinen, \& Forssell, 2008; Shantha, et al., 1994). Briefly, $0.4 \mathrm{~g} \mathrm{BaCl}_{2} \cdot 2 \mathrm{H}_{2} \mathrm{O}$ was dissolved in $50 \mathrm{ml}$ of distilled water. Separately, a ferrous solution was prepared by dissolving $0.5 \mathrm{~g}$ of $\mathrm{FeSO}_{4} \cdot 7 \mathrm{H}_{2} \mathrm{O}$ in $50 \mathrm{ml}$ of distilled water. The barium solution was slowly added to the ferrous one under magnetic stirring, then $2 \mathrm{ml}$ $\mathrm{HCl} 10 \mathrm{~N}$ were added. The $\mathrm{BaSO}_{4}$ precipitate was filtered to obtain a clear $\mathrm{FeCl}_{2}$ solution, which was stored in an opaque flask. Freshly prepared $\mathrm{FeCl}_{2}$ solution was used in each procedure. To prepare the complexing agent, $30 \mathrm{~g}$ of $\mathrm{NH}_{4} \mathrm{SCN}$ were dissolved in $100 \mathrm{ml}$ of distilled water.

Table 1. Storage conditions used for the oxidative stability studies

\begin{tabular}{|c|c|c|c|c|}
\hline Test & $\begin{array}{c}\text { RH } \\
(\%)\end{array}$ & $\begin{array}{c}\text { Temperature } \\
\left({ }^{\circ} \mathbf{C}\right)\end{array}$ & Environment & Storing conditions \\
\hline 1 & 0 & 5 & Air and darkness & Fridge \\
\hline 2 & 0 & 23 & Air and light & Dryness \\
\hline 3 & 65 & 23 & $\begin{array}{c}\text { Vacuum and } \\
\text { darkness }\end{array}$ & Ambient \\
\hline
\end{tabular}

To determine PV of the neat DHA-enriched fish oil, a $20 \mathrm{mg}$ of the free oil was diluted into $1 \mathrm{ml}$ of iso-octane using a vortex stirrer for $5 \mathrm{~s}$. In the case of the zein capsules, a 20 mg sample was completely dissolved in $1 \mathrm{ml}$ of ethanol $85 \mathrm{wt} .-\%$ in a vortex in order to have available for testing all the oil contained inside the capsules. An aliquote of $1 \mathrm{ml}$ isooctane was added to this solution, vortexed again, and the organic phase containing the oil was removed for further analysis. After that, an aliquot of $100 \mu 1$ of the oil solutions 
and $100 \mu 1 \mathrm{NH}_{4} \mathrm{SCN}$ were then added to $5 \mathrm{ml}$ ethanol and mixed in the vortex. Finally, $100 \mu \mathrm{FeCl}_{2}$ was added and vortexed again. After $5 \mathrm{~min}$ of reaction, the absorbance was measured at $500 \mathrm{~nm}$ against a blank containing all reagents excepting the sample. PV was calculated using the following equation:

$$
P V=[(A s-A b) \cdot m] /\left(2 \cdot 55.84 \cdot m_{o}\right)
$$

where $A s$ and $A b$ are the absorbance of the test sample and blank, respectively, $m$ is the slope of the calibration curve, $m_{o}$ is the weight sample ( $\mathrm{g}$ of oil), and $55.84 \mathrm{~g} / \mathrm{mol}$ is the atomic weight of iron. Glassware was washed with diluted $\mathrm{HNO}_{3}$ and rinsed with distilled water before use to eliminate any iron contamination. The samples were measured by triplicate.

\subsection{Headspace oxygen volume depletion}

The oxidative stability of DHA-enriched fish oil was compared with the one of the corresponding zein capsules by measuring the headspace oxygen volume depletion over time at room conditions, i.e. $23^{\circ} \mathrm{C}$ and $40 \%$ RH. For this purpose, a multi-channel oxygen meter OXY-4 mini purchased from PreSens (Regensburg, Germany) was used. Samples of $1.5 \mathrm{~g}$ of fresh DHA-enriched fish oil and its equivalent quantity of zein/DHA enrichedfish oil were placed inside a 100-ml Schleck flasks in which 5-mm spot sensors were previously attached. The non-destructive assays involved the online monitoring of the headspace oxygen using fluorescence decay based on ASTM F2714-08(2013) - Standard Test Method for Oxygen Headspace Analysis of Packages Using Fluorescent Decay. Values were taken for $100 \mathrm{~h}$ and normalized to the initial oxygen volume. The measurements were done in duplicate.

\subsection{Organoleptic test}

Organoleptic tests were performed to estimate the impact of adding zein/DHA-enriched fish oil, compared to neat DHA-enriched fish oil, to a reconstituted milk that was used a food model. The reconstituted milk was prepared by dissolving $25 \mathrm{~g}$ of skimmed powder milk in $130 \mathrm{ml}$ of bottled drinking water. The enriched reconstituted milk samples were prepared by adding $37.5 \mathrm{mg}$ of free DHA-enriched fish oil or $75 \mathrm{mg}$ of zein capsules with DHA-enriched fish oil to $25 \mathrm{~g}$ of skimmed powder milk and $130 \mathrm{ml}$ of bottled drinking 
water. All preparations were stirred with a cooking spoon until complete homogenization. The organoleptic tests were then performed with the freshly prepared capsules ( $\mathrm{t}=0$ day) and 45 days. Overall fishiness attributes, including taste, odor, flavor, and appearance, were evaluated for each sample by six trained panelists from the IATA-CSIC against a reference sample consisting on a reconstituted milk without DHA-enriched fish oil. A 5point hedonic scale was used to score the samples attributes following next attributes: $(0)$ no difference against reference; (1) little difference against reference; (3) clear difference against reference; (5) big difference against reference. The test data were evaluated through analysis of variance (ANOVA) using STATGRAPHICS Centurion XVI v 16.1.03 from StatPoint Technologies, Inc. (Warrenton, VA, USA). Fisher's least significant difference (LSD) was used at the 95\% confidence level $(p<0.05)$. Mean values and standard deviations were also calculated.

\section{RESULTS AND DISCUSSION}

\subsection{Morphology}

The collected zein/DHA-enriched fish oil capsules were analyzed by SEM using a relative low voltage in order to avoid any degradation of particles during observation. Their morphology is shown in Figure 1. The neat zein capsules presented an irregular shape based on a rough-like surface with a mean particle size of $3.7 \pm 1.8 \mu \mathrm{m}$ (see Figure 1a). The incorporation of DHA-enriched fish oil into the zein matrix led to structures with a similar morphology but smaller in size. Thus, the mean particle size was reduced to $1.4 \pm$ $0.8 \mu \mathrm{m}$ (Figure 1b). This change in the capsule morphology can be related to the intrinsic emulsifying effect provided by zein (Filippidi, Patel, Bouwens, Voudouris, \& Velikov, 2014), which could reduce the fish oil droplets in the zein solution for EAPG. A similar effect was observed for electrosprayed dextran capsules loaded with fish oil (GarcíaMoreno, et al., 2017).

It is also worthy to mention that the here-observed morphology differs from the one previously reported for conventional electrosprayed zein particles, where smaller semispherical shrunk submicron capsules, ranging from 175 to $900 \mathrm{~nm}$, were obtained depending on the biopolymer concentration and process parameters (Gomez-Estaca, Balaguer, Gavara, \& Hernandez-Munoz, 2012; Torres-Giner, et al., 2010; Zhang, et al., 2016). Additionally, the formation of fiber-like structures was not observed during EAPG process, which can be related to the relatively low zein concentration used in the solution 
321 (Torres-Giner, Gimenez, \& Lagaron, 2008). The morphology of the here-obtained zein/DHA-enriched fish oil capsules was, however, similar but smaller in size, i.e. 2-3 $\mu \mathrm{m}$, than the zein capsules containing nisin obtained by SD in the work carried out by Xiao, Davidson, \& Zhong (2011). This is related to the fact the elongation process exerted by the electrohydrodynamic forces is accomplished via a contactless scheme, which yields very efficient solvent removal and lower particle sizes (Torres-Giner, et al., 2016).
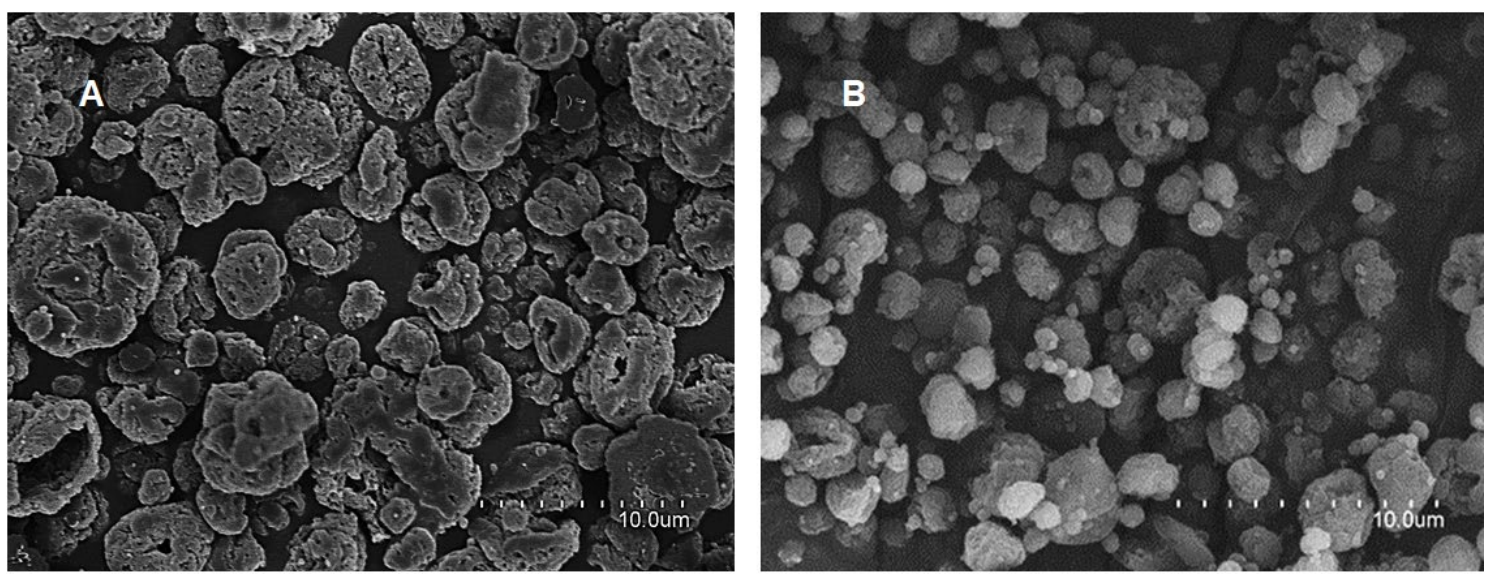

Figure 1. Scanning electron microscopy (SEM) images of: (a) Neat zein microcapsules; (b) Zein/docosahexaenoic acid (DHA)-enriched fish oil microcapsules. Scale markers are $10 \mu \mathrm{m}$.

The optical images taken at bright field were compared to the corresponding ones using a cyan fluorescent filter, which are displayed in Figure 2. Based on the fact that zein is a fluorophore material, while DHA does not exhibit this property (Fernandez, TorresGiner, \& Lagaron, 2009; Gomez-Estaca, et al., 2012; Torres-Giner, et al., 2010), fluorescence microscopy was chosen for evaluate the distribution of the DHA-enrich fish oil in the zein microcapsules. In Figure 2a one can observe that the irregular morphology of the neat zein capsules emitted intensely in the fluorescent field. Structures with strong fluorescence but with somewhat lower emission and less defined shapes, i.e. particles with fuzzy edges, were also observed for the zein/DHA-enriched fish oil microcapsules, as shown in Figure 2b. This suggests that the fish oil was successfully entrapped within the zein matrix, which is in agreement with some previous studies concerning bioactivecontaining zein structures prepared by EHDP (Fernandez, et al., 2009; Torres-Giner, et al., 2010). 

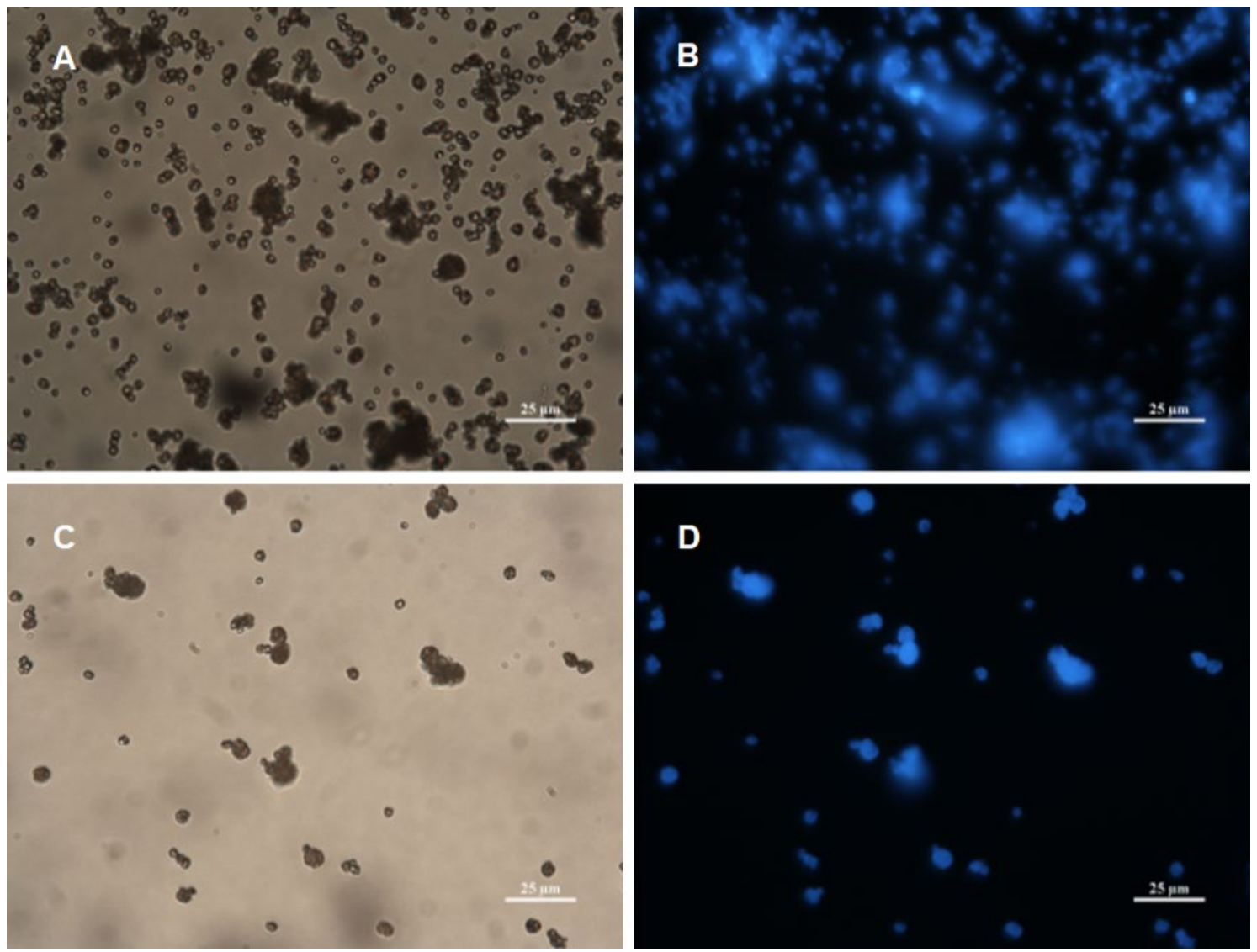

Figure 2. Optical microscopy images of: Neat zein microcapsules under visible (a) and fluorescent light (b); Zein/docosahexaenoic acid (DHA)-enriched fish oil microcapsules under visible (c) and fluorescent light (d). Scale markers are $25 \mu \mathrm{m}$.

\subsection{Encapsulation efficiency}

351 A mild oil extraction method was applied to quantify the free oil and/or easily extractable oil from inside the capsule by UV spectroscopy. The encapsulation efficiency in the zein microcapsules was found to be of $84 \pm 1 \%$. This indicates that a large amount of DHAenriched fish oil is effectively protected by the zein wall avoiding oxidation that could cause undesirable changes in terms of nutritional, organoleptic, and bulk properties. In this regard, efficiency of DHA microencapsulation by conventional SD process has been reported in the 57-98\% range for water-soluble wall materials (Bakry, et al., 2016), though no specific values for the non-water soluble zein have been reported yet. The encapsulation efficiency of the here-prepared zein microcapsules obtained by EAPG is within the same range as electrosprayed fish oil capsules made of water soluble WPI, dextran, and pullulan, which presented yields between 69-85\% (García-Moreno, et al., 2017; Wang, et al., 2016). However, electrospun zein-fish oil fibers obtained by single, 
coaxial, and emulsion electrospinning have been reported to yield efficiencies of approximately 95\%, 97\%, and 95\%, respectively (García-Moreno, et al., 2016; Moomand, et al., 2014; Yang, Feng, Wen, Zong, Lou, \& Wu, 2017). This result can be related to the lower content of both fish oil and/or DHA within the fish oil, to the significantly different morphology, and to the fibers forming a continuous more efficient barrier than particles.

\subsection{Oxidative stability}

PV is a measure of primary oxidation of fatty acids, by which the corresponding fatty acids hydroperoxides are quantified. In the specific case of fish oils, one common drawback of their use and formulation is related to their intrinsic hydrophobicity and the high rate at which these oxidize. For this reason, both stabilization in aqueous medium and protection against external stimuli that trigger deterioration is required.

The zein/DHA-enriched fish oil microcapsules were exposed to different storing conditions, as shown in previous Table 1, in terms of RH, i.e. $0 \%$ and $65 \%$, temperature, i.e. $5^{\circ} \mathrm{C}$ and $23{ }^{\circ} \mathrm{C}$, and environment, i.e. air and vacuum as well as light and darkness. This study was carried out to compare the oxidative stability of the encapsulated versus the non-encapsulated DHA-enriched fish oil and also to ascertain their storage stability under different conditions. As it can be observed in Figure 3, all samples presented an initial PV of $\sim 1.5 \mathrm{meq} / \mathrm{kg}$ oil, which is a relatively low value, taking into account that the Global Organization for EPA and DHA Omega-3s (GOED) sets a limit for DHA oils of $5 \mathrm{meq} / \mathrm{kg}$ (GOED, 2015). It can be considered that, even though the encapsulation process was carried out using air flow at room temperature, DHA oxidation was limited by the continuous bubbling of nitrogen to the zein solution during the process as well as the frequent withdrawal of the product from the collector and subsequent storage under vacuum. Figure 3 also indicates that PV increased in all samples because of the primary oxidation of DHA. However, PV then decreased as the formed hydroperoxides decomposed and secondary oxidation products arose (presumably aldehydes, ketones, and alcohols of distinct chain lengths and degrees of unsaturation) (Pereira, et al., 2014). In the free DHA-enriched fish oil, the hydroperoxides concentration was significantly higher than that in the encapsulated zein microcapsules, being the lowest PV observed for the samples tested under vacuum. This indicates that the DHA contained in the zein microcapsules was less prone to oxidative degradation. These results correlate well with 
396 the estimated high encapsulation efficiency described above. Similar results were 397 obtained by Partanen, et al. (2008) for the encapsulation of flaxseed oil in WPI by SD 398 after 3 weeks at $40^{\circ} \mathrm{C}$.

399 In relation to the different conditions here-studied, one can observe in Figures 3a and 3b 400 that the free and encapsulated DHA-enriched fish oils presented maximum PVs, of 401 approximately 43 and 19-22 meq/kg oil, respectively, when exposed to both tested 402 temperatures, i.e. $5^{\circ} \mathrm{C}$ and $23^{\circ} \mathrm{C}$, in the presence of air at $0 \% \mathrm{RH}$. However, as expected, 403 the maximum PV was achieved earlier in time at the highest tested temperature, i.e. $23^{\circ} \mathrm{C}$. 404 Thus, for the encapsulated DHA-enriched fish oil, the secondary oxidative reactions 405 started approximately 7 days later at $5^{\circ} \mathrm{C}$ than at $23^{\circ} \mathrm{C}$. In Figure $3 \mathbf{c}$ one can observe that 406 when samples were exposed to $23^{\circ} \mathrm{C}$ in the absence of oxygen but in the presence of a 407 higher humidity, i.e. $65 \% \mathrm{RH}$, and protected from light, PV exhibited remarkably lower 408 values for both the free and encapsulated DHA-enriched fish oil. Interestingly, at these 409 conditions, the free and encapsulated DHA-enriched fish oil showed PVs of 410 approximately 18 and below $2 \mathrm{meq} / \mathrm{kg}$ oil, respectively, which means that oxygen is, as 411 expected, the most influencing factor in DHA oxidation as compared to both temperature 412 and humidity. 
A

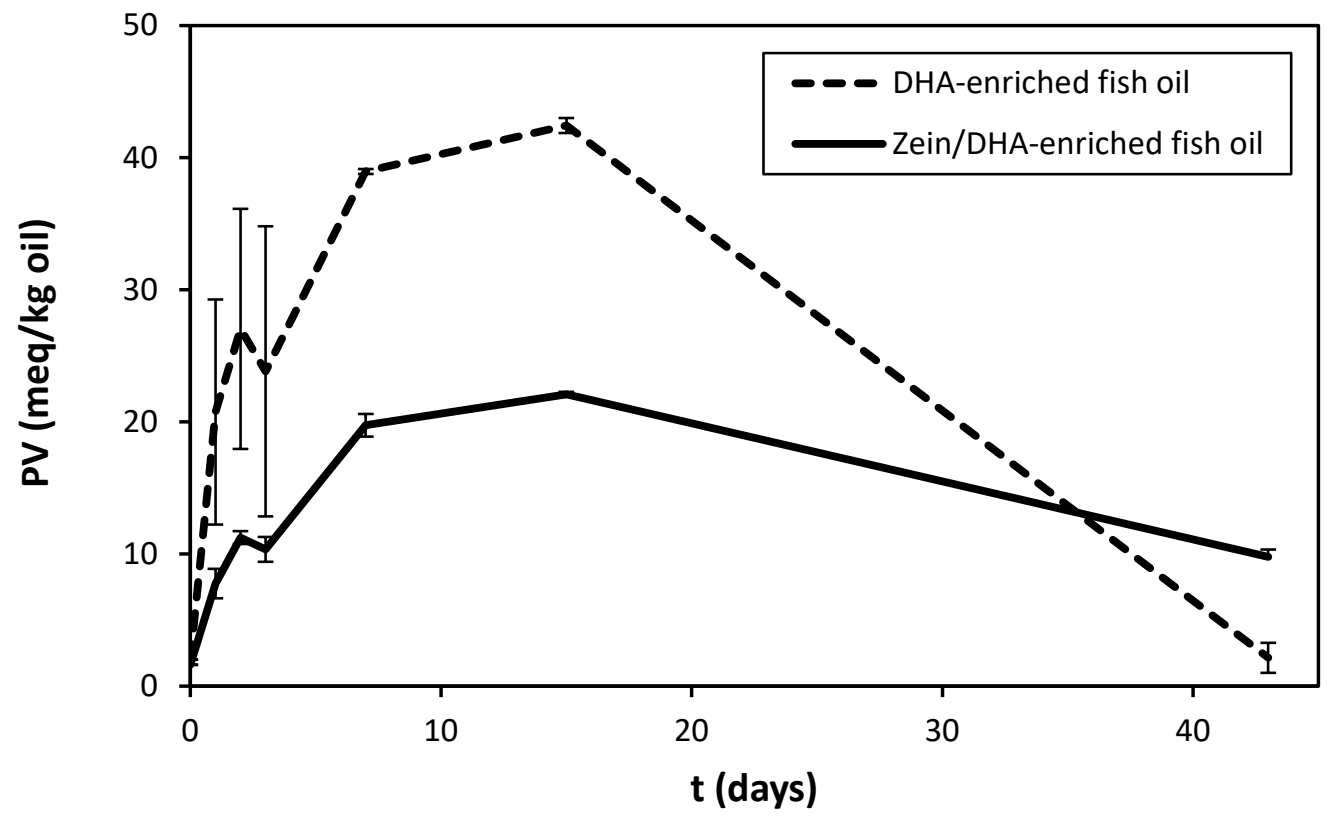

413

414

415 
B

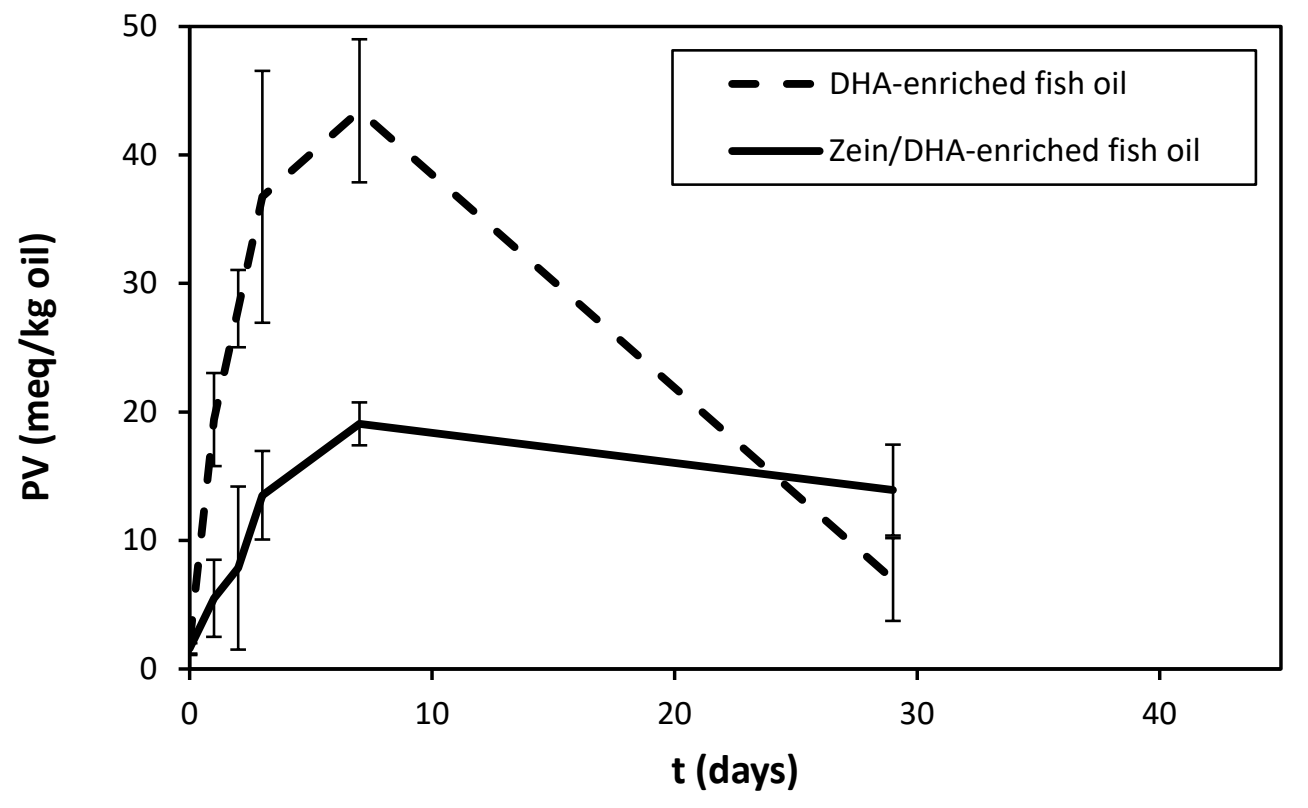

416

417 
C

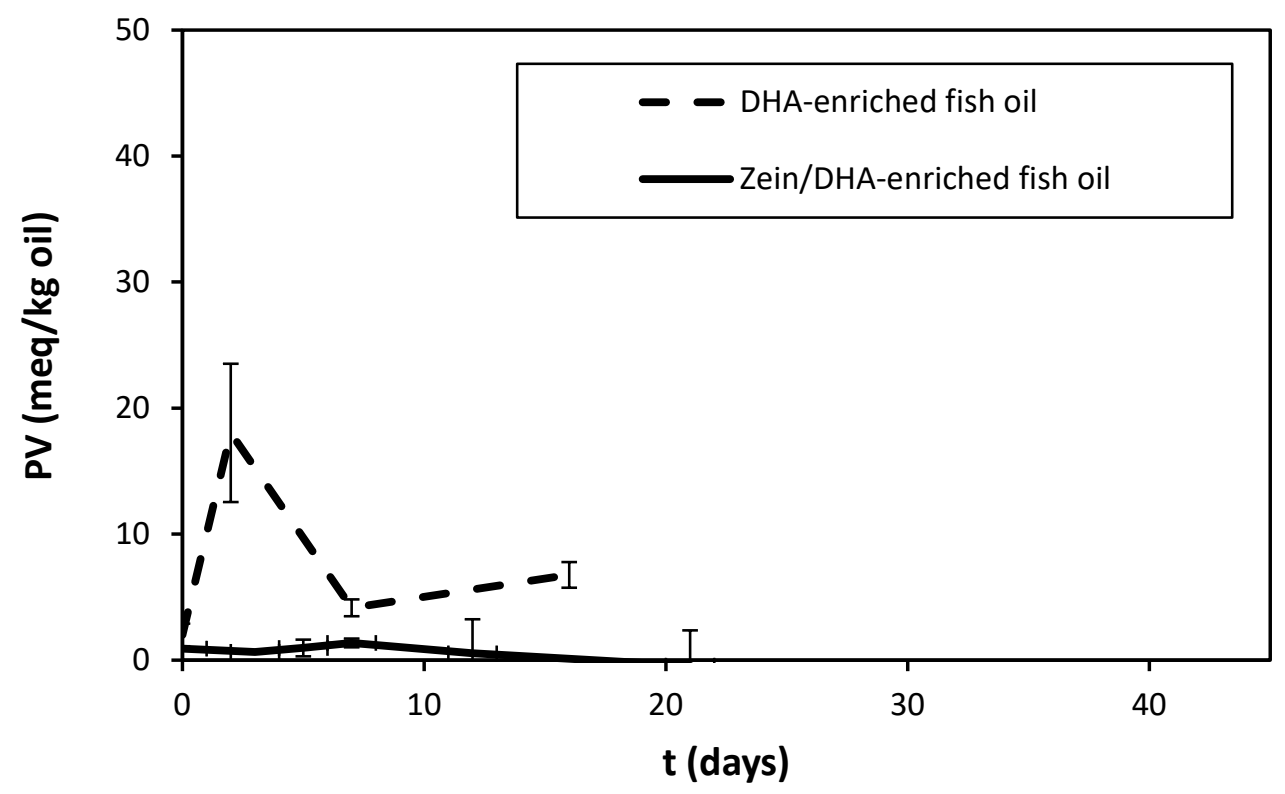

420 Figure 3. Comparative trends of the peroxide values (PV), expressed as meq $/ \mathrm{kg}$ oil, 421 between the free and encapsulated docosahexaenoic acid (DHA)-enriched fish oil in zein 422 microcapsules at different conditions: (a) $5^{\circ} \mathrm{C}$, air and darkness, and $0 \% \mathrm{HR}$; (b) $23^{\circ} \mathrm{C}$, 423 air and light, and $0 \% \mathrm{RH}$; (c) $23^{\circ} \mathrm{C}$, vacuum and darkness, and $65 \% \mathrm{RH}$.

\subsection{Headspace oxygen depletion}

427 Figure 4 shows the percentage of headspace oxygen depletion, determined by the 428 fluorescence decay method, for an equivalent amount of free and encapsulated DHA- 
429 enriched fish oil. This was performed under room temperature conditions, i.e. $40 \% \mathrm{RH}$ 430 and $23^{\circ} \mathrm{C}$. This was achieved by means of spot sensors in a sealed space filler with air, a 431 technique that has been widely used to determine oxygen permeability and oxygen 432 scavenging in sealed packaging materials (Busolo \& Lagaron, 2012). However, in the 433 present study it was applied to monitor the oxidation of a DHA-enriched oil in order to 434 assess the efficiency against oxygen penetration in an encapsulate. From Figure 4, one 435 can observe that the free DHA-enriched fish oil sample oxidized significantly faster than 436 the one encapsulated in the zein microcapsules produced by EAPG. At the end of the test, 437 i.e. after $100 \mathrm{~h}$, the free DHA-enriched fish oil consumed $27 \%$ of the headspace oxygen 438 volume, while this value reached only $\sim 8 \%$ in the encapsulated sample. Interestingly, 439 while the free DHA-enriched fish oil followed a monotonic linear decrease in oxygen 440 depletion, the encapsulated DHA-enriched fish oil decreased with a lower slope at the 441 beginning and then this decrease becomes arrested with a tendency to reach a plateau. In 442 this regard, it is worthy to mention that zein acts as a high barrier matrix to oxygen when 443 dry (Tihminlioglu, Atik, \& Özen, 2010). Thus, the hydrocolloid is able to block oxygen 444 molecules diffusion and, thus, it strongly contributes to preventing DHA oxidation. 445 However, under ambient RH conditions, such as the ones in this test, protein plasticization 446 is expected to occur to some extent, hence leading to faster penetration of oxygen and 447 oxidation.

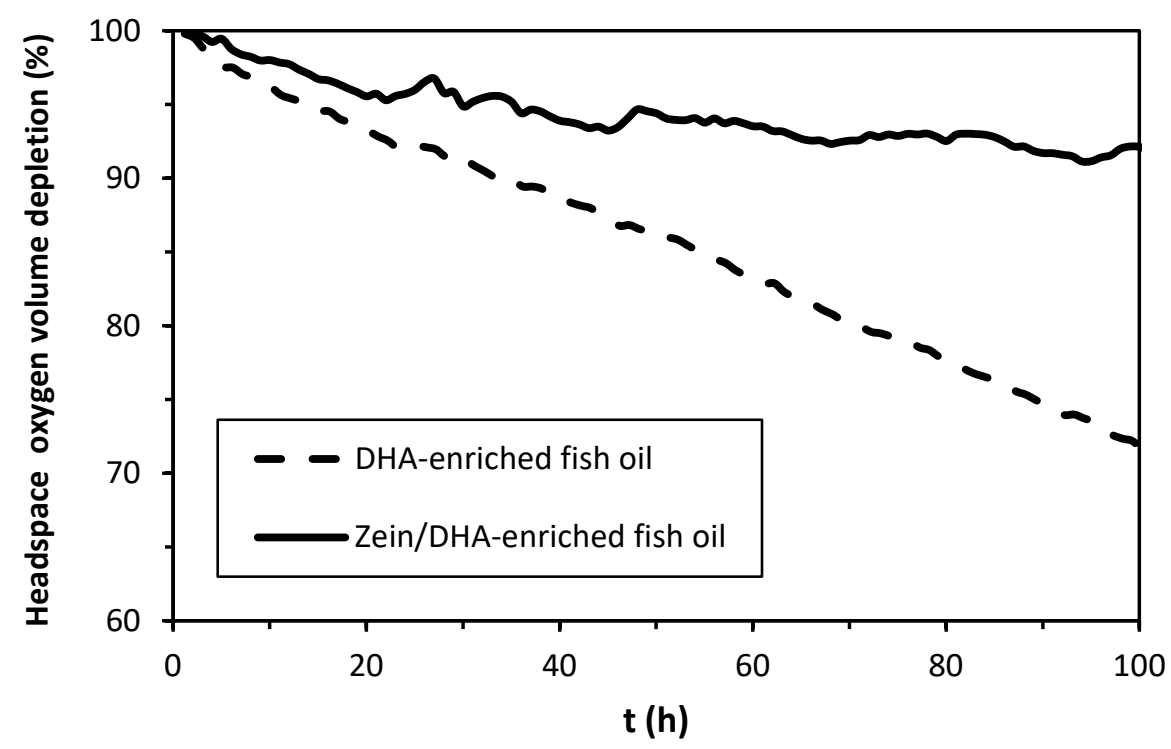


449 Figure 4. Evolution of the percentage of headspace oxygen volume over time for the free and encapsulated docosahexaenoic acid (DHA)-enriched fish oil in zein microcapsules. Typical deviation among specimens was less than $2 \%$.

452

\subsection{Organoleptic properties}

454 Due to their easy-to-handle properties, high encapsulation efficiency, and improved oxidation stability, it is expected that the zein/DHA-enriched fish oil microcapsules prepared by EAPG should reduce the formation of oxidized off-flavors. Hence, these novel capsules can be used to formulate fortified food products, such as milk powder, minimizing the presence of undesirable fishiness flavors. In this context, the organoleptic characteristics of food preparations are the ones ultimately dictating acceptance into specific applications. However, it has been found a low correlation between analytical and human sensory for microencapsulated fish oil due to complexity and own sensory properties of each encapsulation system (C. Barrow, et al., 2007). Therefore, it is currently unclear whether instruments can replace sensory panels for better accuracy in sensory tests.

During samples preparation, it was noticed that the zein/DHA-enriched fish oil microcapsules were readily dispersed after vigorous spoon agitation in the milk preparation, thus homogeneous non-lumpy solutions were achieved (same as reference). However, when preparing the fortified milk sample with the free DHA-enriched fish oil, even after vigorous spoon agitation, some tiny oil drops remained on the milk surface. As it can be seen in Figure 5, when the panelists tested the fortified milk preparation containing the fresh zein/DHA-enriched fish oil microcapsules, they found little difference against the blank reference, i.e. the unfortified reconstituted milk. However,

473 they perceived a clear difference versus the test sample containing the fresh free DHAenriched fish oil.

The fortified milk samples were prepared again using both free and encapsulated DHAenriched fish oil that was stored at $-1{ }^{\circ} \mathrm{C}$ for 45 days under vacuum and protected from

477 light. In the second test, the panelists maintained the score for the sample containing the free DHA-enriched fish oil since they still perceived similar unpleasant properties in this milk sample. In the same way, the fortified milk prepared with the zein/DHA-enriched fish oil microcapsules were, once more, valued as having little difference in relation to the reference. The observations provided by the panelists can be then mainly related to 
both the presence of the characteristic unpleasant fishiness odor and flavor of the free

483 DHA-enriched fish oil as well as to the undesirable appearance of oil drops in the milk surface. The absence of difference after storage time in the milk samples enriched with zein microcapsules can be attributed to the high encapsulation efficiency achieved by EAPG. This correlates well with the previous headspace oxygen volume depletion test.

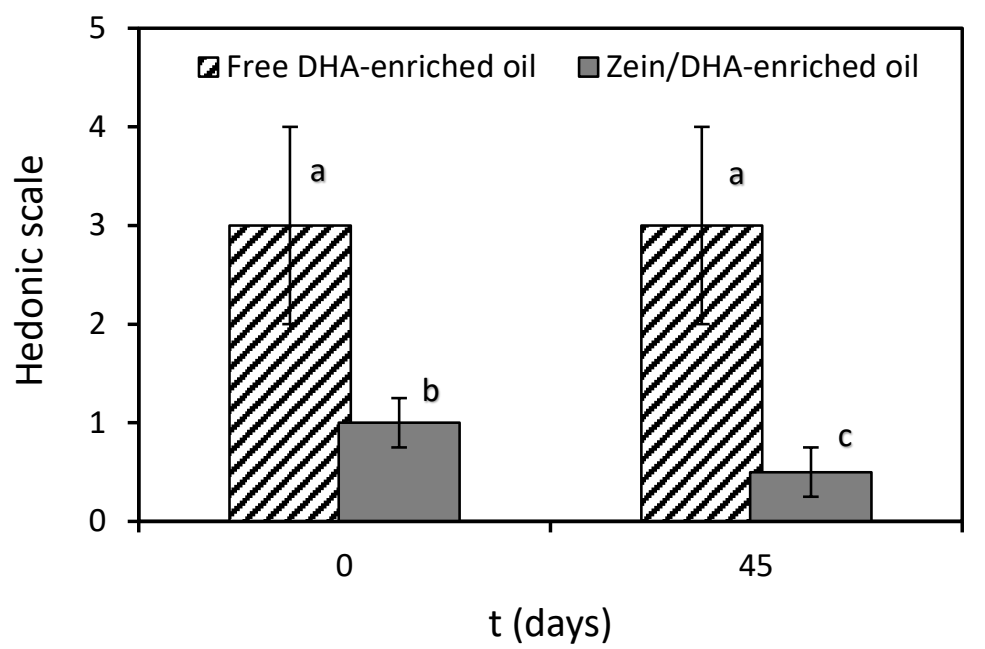

Figure 5. Panelists score of reconstituted milk samples containing free and encapsulated docosahexaenoic acid (DHA)-enriched fish oil in zein microcapsules, both fresh ( $\mathrm{t}=0$ day) and after 45 days of production, being stored at $-1{ }^{\circ} \mathrm{C}$, vacuum, and protected from light. Different letters indicate significant differences among samples $(\mathrm{p}<0.05)$.

\section{CONCLUSIONS}

Zein/DHA-enriched fish oil flowable microcapsules were obtained, for the first time, by the innovative EAPG technique. This is based on a combination of high electric field with pneumatic spraying. By this novel approach, it was possible to encapsulate DHAenriched fish oil in micrometric semi-spherical zein capsules, with mean sizes of $1.4 \mu \mathrm{m}$, showing an encapsulation efficiency of $84 \pm 1 \%$. In addition, DHA, a valuable nutraceutical which rapidly oxidizes, was successfully stabilized in the zein microcapsules due to the low temperature and fast evaporation characteristics of the EAPG process. In particular, the highest stability was observed for the capsules stored under vacuum at $23{ }^{\circ} \mathrm{C}, 56 \% \mathrm{RH}$, and protected from light, as determined by oxidative stability assays. Finally, sensory tests carried out by independent panelists showed that the enrichment of zein/DHA-enriched fish oil microcapsules to a reconstituted milk 
preparation considerably reduced the organoleptic impact in comparison to the free DHAenriched fish oil. In addition, similar organoleptic properties were reported after 45 days of storage under firm storage conditions. The obtained results indicate that EAPG processing could become a very promising technique for the microencapsulation of sensitive materials, such as nutraceuticals, which can be used thereafter to develop fortified food products.

\section{ACKNOWLEDGEMENTS}

The authors would like to thank the Spanish Ministry of Economy and Competitiveness (MINECO) project AGL2015-63855-C2-1-R and to the H2020 EU project YPACK (reference number 773872) for funding.

\section{REFERENCES}

Aghbashlo, M., Mobli, H., Madadlou, A., \& Rafiee, S. (2012). The correlation of wall material composition with flow characteristics and encapsulation behavior of fish oil emulsion. Food Research International, 49(1), 379-388.

Anwar, S. H., \& Kunz, B. (2011). The influence of drying methods on the stabilization of fish oil microcapsules: Comparison of spray granulation, spray drying, and freeze drying. Journal of Food Engineering, 105(2), 367-378.

Anwar, S. H., Weissbrodt, J., \& Kunz, B. (2010). Microencapsulation of Fish Oil by Spray Granulation and Fluid Bed Film Coating. Journal of Food Science, 75(6), E359-E371.

Arbabi, L., Baharuldin, M. T. H., Moklas, M. A. M., Fakurazi, S., \& Muhammad, S. I. (2014). Antidepressant-like effects of omega-3 fatty acids in postpartum model of depression in rats. Behavioural Brain Research, 271, 65-71.

Ayelén Vélez, M., Cristina Perotti, M., Santiago, L., María Gennaro, A., \& Hynes, E. (2017). 6 Bioactive compounds delivery using nanotechnology: design and applications in dairy food A2 - Grumezescu, Alexandru Mihai. In Nutrient Delivery (pp. 221-250): Academic Press.

Bakry, A. M., Abbas, S., Ali, B., Majeed, H., Abouelwafa, M. Y., Mousa, A., \& Liang, L. (2016). Microencapsulation of Oils: A Comprehensive Review of Benefits, Techniques, and Applications. Comprehensive Reviews in Food Science and Food Safety, 15(1), 143-182.

Barrow, C., Van Diepen, C., Perrie, C., Curtis, J., Jin, Y., \& Zhang, W. (2007). Microencapsulation of Marine Lipids as a Vehicle for Functional Food Delivery. In Marine Nutraceuticals and Functional Foods (pp. 115-154): CRC Press.

Barrow, C. J., Wang, B., Adhikari, B., \& Liu, H. (2013). 6 - Spray drying and encapsulation of omega-3 oils. In Food Enrichment with Omega-3 Fatty Acids (pp. 194-225): Woodhead Publishing.

Busolo, M. A., \& Lagaron, J. M. (2012). Oxygen scavenging polyolefin nanocomposite films containing an iron modified kaolinite of interest in active food packaging applications. Innovative Food Science \& Emerging Technologies, 16(Supplement C), 211-217.

Chang, M.-W., Stride, E., \& Edirisinghe, M. (2010). Controlling the thickness of hollow polymeric microspheres prepared by electrohydrodynamic atomization. Journal of The Royal Society Interface, 7(Suppl 4), S451-S460. 
Chen, W., Wang, H., Zhang, K., Gao, F., Chen, S., \& Li, D. (2016). Physicochemical Properties and Storage Stability of Microencapsulated DHA-Rich Oil with Different Wall Materials. Applied Biochemistry and Biotechnology, 179(7), 1129-1142.

Eltayeb, M., Stride, E., Edirisinghe, M., \& Harker, A. (2016). Electrosprayed nanoparticle delivery system for controlled release. Materials Science and Engineering: C, 66, 138146.

Encina, C., Vergara, C., Giménez, B., Oyarzún-Ampuero, F., \& Robert, P. (2016). Conventional spray-drying and future trends for the microencapsulation of fish oil. Trends in Food Science \& Technology, 56, 46-60.

Fernandez, A., Torres-Giner, S., \& Lagaron, J. M. (2009). Novel route to stabilization of bioactive antioxidants by encapsulation in electrospun fibers of zein prolamine. Food Hydrocolloids, 23(5), 1427-1432.

Filippidi, E., Patel, A. R., Bouwens, E. C. M., Voudouris, P., \& Velikov, K. P. (2014). All-Natural Oil-Filled Microcapsules from Water-Insoluble Proteins. Advanced Functional Materials, 24(38), 5962-5968.

Ganesan, B., Brothersen, C., \& McMahon, D. J. (2014). Fortification of Foods with Omega-3 Polyunsaturated Fatty Acids. Critical Reviews in Food Science and Nutrition, 54(1), 98114.

García-Moreno, P. J., Özdemir, N., Stephansen, K., Mateiu, R. V., Echegoyen, Y., Lagaron, J. M., Chronakis, I. S., \& Jacobsen, C. (2017). Development of carbohydrate-based nanomicrostructures loaded with fish oil by using electrohydrodynamic processing. Food Hydrocolloids, 69, 273-285.

García-Moreno, P. J., Stephansen, K., van der Kruijs, J., Guadix, A., Guadix, E. M., Chronakis, I. S., \& Jacobsen, C. (2016). Encapsulation of fish oil in nanofibers by emulsion electrospinning: Physical characterization and oxidative stability. Journal of Food Engineering, 183, 39-49.

GOED. (2015). Oxidation in Omega-3 Oils: An Overview. A White Paper Prepared by the Global Organization for EPA and DHA Omega-3s and the Council for Responsible Nutrition. In GOED Voluntary Monograph (pp. 2).

Gomez-Estaca, J., Balaguer, M. P., Gavara, R., \& Hernandez-Munoz, P. (2012). Formation of zein nanoparticles by electrohydrodynamic atomization: Effect of the main processing variables and suitability for encapsulating the food coloring and active ingredient curcumin. Food Hydrocolloids, 28(1), 82-91.

Heinzelmann, K., Franke, K., Jensen, B., \& Haahr, A.-M. (2000). Protection of fish oil from oxidation by microencapsulation using freeze-drying techniques. European Journal of Lipid Science and Technology, 102(2), 114-121.

Hogan, S. A., O'Riordan, E. D., \& O'Sullivan, M. (2003). Microencapsulation and oxidative stability of spray-dried fish oil emulsions. Journal of Microencapsulation, 20(5), 675688.

Hong, X., Mahalingam, S., \& Edirisinghe, M. (2017). Simultaneous Application of PressureInfusion-Gyration to Generate Polymeric Nanofibers. Macromolecular Materials and Engineering, 302(6), 1600564.

Krokida, M. (2017). Thermal and Nonthermal Encapsulation Methods. Boca Raton, Florida, USA: CRC Press.

Lagaron, J. M., Castro, S., Galan, D., \& Valle, J. M. (2017). Installation and procedure of industrial encapsulation of thermolabile substances. P201631725.

Lopez-Huertas, E. (2010). Health effects of oleic acid and long chain omega-3 fatty acids (EPA and DHA) enriched milks. A review of intervention studies. Pharmacological Research, 61(3), 200-207.

Moomand, K., \& Lim, L.-T. (2014). Oxidative stability of encapsulated fish oil in electrospun zein fibres. Food Research International, 62, 523-532.

Park, J.-M., Kwon, S.-H., Han, Y.-M., Hahm, K.-B., \& Kim, E.-H. (2013). Omega-3 Polyunsaturated Fatty Acids as Potential Chemopreventive Agent for Gastrointestinal Cancer. Journal of Cancer Prevention, 18(3), 201-208. 
Partanen, R., Raula, J., Seppänen, R., Buchert, J., Kauppinen, E., \& Forssell, P. (2008). Effect of Relative Humidity on Oxidation of Flaxseed Oil in Spray Dried Whey Protein Emulsions. Journal of Agricultural and Food Chemistry, 56(14), 5717-5722.

Pereira, D. M., Valentão, P., \& Andrade, P. B. (2014). Nano- and Microdelivery Systems for Marine Bioactive Lipids. Marine Drugs, 12(12), 6014-6027.

Prieto, C., \& Calvo, L. (2017). The encapsulation of low viscosity omega-3 rich fish oil in polycaprolactone by supercritical fluid extraction of emulsions. The Journal of Supercritical Fluids, 128, 227-234.

Ruxton, C. H. S., Reed, S. C., Simpson, M. J. A., \& Millington, K. J. (2004). The health benefits of omega-3 polyunsaturated fatty acids: a review of the evidence. Journal of Human Nutrition and Dietetics, 17(5), 449-459.

Shams, T., Parhizkar, M., Illangakoon, U. E., Orlu, M., \& Edirisinghe, M. (2017). Core/shell microencapsulation of indomethacin/paracetamol by co-axial electrohydrodynamic atomization. Materials \& Design, 136, 204-213.

Shantha, N. C., \& Decker, E. A. (1994). Rapid, sensitive, iron-based spectrophotometric methods for determination of peroxide values of food lipids. J AOAC Int, 77(2), 421-424.

Siriwardhana, N., Kalupahana, N. S., \& Moustaid-Moussa, N. (2012). Health Benefits of n-3 Polyunsaturated Fatty Acids. Advances in Food and Nutrition Research, 65, 211-222.

Sun, G. Y., Simonyi, A., Fritsche, K. L., Chuang, D. Y., Hannink, M., Gu, Z., Greenlief, C. M., Yao, J. K., Lee, J. C., \& Beversdorf, D. Q. (2017). Docosahexaenoic acid (DHA): An essential nutrient and a nutraceutical for brain health and diseases. Prostaglandins, Leukotrienes and Essential Fatty Acids (PLEFA).

Tapia-Hernández, J. A., Torres-Chávez, P. I., Ramírez-Wong, B., Rascón-Chu, A., PlascenciaJatomea, M., Barreras-Urbina, C. G., Rangel-Vázquez, N. A., \& Rodríguez-Félix, F. (2015). Micro- and Nanoparticles by Electrospray: Advances and Applications in Foods. Journal of Agricultural and Food Chemistry, 63(19), 4699-4707.

Tihminlioglu, F., Atik, İ. D., \& Özen, B. (2010). Water vapor and oxygen-barrier performance of corn-zein coated polypropylene films. Journal of Food Engineering, 96(3), 342-347.

Torres-Giner, S. (2011). Electrospun nanofibers for food packaging applications. In Multifunctional and Nanoreinforced Polymers for Food Packaging (pp. 108-125).

Torres-Giner, S., Gimenez, E., \& Lagaron, J. M. (2008). Characterization of the morphology and thermal properties of Zein Prolamine nanostructures obtained by electrospinning. Food Hydrocolloids, 22(4), 601-614.

Torres-Giner, S., Martinez-Abad, A., Ocio, M. J., \& Lagaron, J. M. (2010). Stabilization of a Nutraceutical Omega-3 Fatty Acid by Encapsulation in Ultrathin Electrosprayed Zein Prolamine. Journal of Food Science, 75(6), N69-N79.

Torres-Giner, S., Pérez-Masiá, R., \& Lagaron, J. M. (2016). A review on electrospun polymer nanostructures as advanced bioactive platforms. Polymer Engineering \& Science, 56(5), 500-527.

Vaughan, V. C., Hassing, M. R., \& Lewandowski, P. A. (2013). Marine polyunsaturated fatty acids and cancer therapy. British Journal of Cancer, 108(3), 486-492.

Wang, Y., Liu, W., Chen, X. D., \& Selomulya, C. (2016). Micro-encapsulation and stabilization of DHA containing fish oil in protein-based emulsion through mono-disperse droplet spray dryer. Journal of Food Engineering, 175, 74-84.

Woods, J., \& Mellon, M. (1941). Thiocyanate Method for Iron: A Spectrophotometric Study. Industrial \& Engineering Chemistry Analytical Edition, 13(8), 551-554.

Xiao, D., Davidson, P. M., \& Zhong, Q. (2011). Release and antilisterial properties of nisin from zein capsules spray-dried at different temperatures. LWT - Food Science and Technology, 44(10), 1977-1985.

Yang, H., Feng, K., Wen, P., Zong, M.-H., Lou, W.-Y., \& Wu, H. (2017). Enhancing oxidative stability of encapsulated fish oil by incorporation of ferulic acid into electrospun zein mat. LWT - Food Science and Technology, 84, 82-90.

Zainal, Z., Longman, A. J., Hurst, S., Duggan, K., Caterson, B., Hughes, C. E., \& Harwood, J. L. (2009). Relative efficacies of omega-3 polyunsaturated fatty acids in reducing expression 
of key proteins in a model system for studying osteoarthritis. Osteoarthritis and Cartilage, 17(7), 896-905.

659 Zeisel, S. H. (1999). Regulation of \&quot;Nutraceuticals\&quot. Science, 285(5435), 1853.

660 Zhang, Y., Cui, L., Li, F., Shi, N., Li, C., Yu, X., Chen, Y., \& Kong, W. (2016). Design, fabrication and biomedical applications of zein-based nano/micro-carrier systems.

662 International Journal of Pharmaceutics, 513(1), 191-210. 